\title{
High density oxygen plasma ashing of CVD-diamond coating with minimum damage to WC (Co) tool substrates
}

\author{
Ersyzario Edo YUNATA*, Tatsuhiko AIZAWA ${ }^{* *}$ and Kazuhisa YAMAUCHI ${ }^{* * *}$ \\ "Department of Regional Environment System, Shibaura Institute of Technology \\ 3-9-14 Shibaura, Minato-City, Tokyo 108-8548, Japan \\ E-mail: na13101@shibaura-it.ac.jp \\ **Department of Engineering and Design, Shibaura Institute of Technology \\ 3-9-14 Shibaura, Minato-City, Tokyo 108-8548, Japan \\ Department of Material Science and Engineering, Shibaura Institute of Technology \\ 3-9-14 Shibaura, Minato-City, Tokyo 108-8548, Japan
}

Received 30 September 2015

\begin{abstract}
Huge amount of CVD-diamond coated milling tools were used for machining of CFRP and CFRTP sheets and blocks in the airplane and automotive industries. Because of chipping and tooth-tip damage in the diamond coatings during those dry machining processes, the tools must be exchanged with new ones to preserve the geometric accuracy in practice. WC (Co) substrate in these used tools had to be recycled to lower the production cost; reliable ashing process was necessary to remove only the used diamond coatings without significant damages even to the tooth tip of substrate. Furthermore, fast-rate ashing became a key to shorten the leading time for exchange of milling and drilling tools. High density oxygen plasma ashing method with use of the hollow cathode device was proposed to remove the used diamond coating with the film thickness of $10 \mu \mathrm{m}$. Both the emissive-light optical spectroscopy and the Langmuir probe method were employed to make quantitative diagnosis on the generated oxygen plasmas. The average ion density increased up to more than $1 \times 10^{17} \mathrm{~m}^{-3}$, higher than the conventional plasma states by one order. Activated oxygen atom had overwhelming population among the generated species in this high plasmas density. This high density oxygen flux was responsible for complete and fast-rate removal of CVD diamond coating; e.g. the average ashing rate turned to be more than $10 \mu \mathrm{m} /$ hour. The short-shank, end-milling tools were employed to describe this ashing behavior with time. Corresponding to the variation of CO-peak intensity in the measured spectra by on-line spectroscopy, the diamond film thickness reduced monotonically with time up to $3.6 \mathrm{ks}$. The removal rate gradually decreased with time in the final stage. Fine tuning of oxygen plasma processing conditions was capable to reduce the damage depth of tool teeth tips down to $1 \mu \mathrm{m}$, significantly less than the standard tolerance of $5 \mu \mathrm{m}$.
\end{abstract}

Key words : CVD-diamond coating, Hollow cathode plasma, Oxygen plasma ashing, Cutting tools, Fast removal rate, Plasma diagnosis

\section{Introduction}

Among several candidate materials, the carbon-based film has been widely utilized as a protective coating material for dry milling tools; e.g. DLC (diamond like-carbon) and CVD-diamond coatings. In fact, various kinds of DLC-coated tools are applied to dry end-milling of aluminum alloys (Zolgharni, et al., 2008). The diamond-coated end-milling tools are also employed for dry milling and drilling of CFRP (carbon fiber reinforced plastics) and CFRTP (carbon fiber reinforced thermo-plastics) structural members and components of aircrafts and automobiles (Hasegawa, 2009).

Owing to their high hardness and engineering endurance, both the DLC- and diamond-coated tool lives were significantly prolonged to reduce the production cost (Aizawa, et al., 2011). However, they often suffer from the 
abrasive wear and chipping. When using the DLC-coated tools in dry, the DLC-coating thickness is gradually reduced by the abrasive wear during dry machining of AA6005 alloys (Gangopadhyay, et al., 2010). The diamond coated tooth suffers from the chipping and loss in tooth-edge geometry during the dry end-milling of CFRP plates (Hasegawa, 2009).

Both DLC and CVD-diamond films are coated onto the WC (Co) tool substrate, which must be recycled by ashing the used DLC- and diamond coatings from WC (Co) and reused as a recoated tool in order to improve their cost-competitiveness in production. In the literature, many ashing processes were reported; e.g. the laser machining (Kurita, et al., 2008), the ion-beam and plasma discharging (Allen, et al,. 2009) and the reactive etching method (Syrkin, et al., 1997). Among them, the oxygen plasma ashing method provides the most rational solution to the perfect removal of carbon-based coatings with minimum damage to WC (Co) tooth shapes of tools. In fact, our developing RF-DC oxygen plasma ashing method works well for perfect ashing of the used DLC coating as well as the metallic interlayers without damage to WC (Co) end-milling tools (Aizawa, et al., 2013; Aizawa, et al., 2014; Aizawa, et al., 2014). In addition, the dry-milling test demonstrates the DLC-recoated tools have the same machinability as the virgin DLC-coated ones; e.g. no defects and chipping were seen even after dry milling by $200 \mathrm{~m}$ in distance and by $240 \mathrm{~cm}^{-3}$ in volume of AA5025 aluminum alloys. However, this ashing method takes long processing time even for partial removal of CVD-diamond coating on the WC (Co) tools; e.g. tedious oxygen ashing for more than 20 hours in commercial (Aizawa, et al., 2014). The above inefficient or tedious ashing behavior comes from too low ion and electron densities to make perfect removal of CVD-diamond films from the WC (Co) tools.

In the present paper, a new hollow cathode device is designed to intensify the oxygen ion and electron densities in the hollow. The quantitative plasma diagnosis is employed to describe the population of activated species as well as the ion and electron density distributions through the hollow tube. Higher oxygen ion density than the conventional plasmas by one order is attained by well set-up of the hollow cathode device. The CVD-diamond coated, short-shank end-milling tools are used and fixed in the optimally set-up of hollow cathode device for ashing experiments. Both the RF-voltage and DC-bias are varied to investigate the optimum condition where the diamond coating is completely ashed away with the minimum loss of WC (Co) tooth-edge geometry and without over-oxidation of WC (Co) substrates. On-line quantitative plasma diagnosis is first employed to describe the ashing process by tracing the CO-peak intensity in time and to terminate the process at the time when the monitored CO-peak intensity becomes minimum. Furthermore, temporal variation of activated oxygen atom peak intensity is utilized to describe the effect of RF-voltage and DC-bias on the oxidation of diamond coatings as well as WC (Co) substrates. SEM as well as optical microscope is used to make microstructural observation on the ashed tooth surface. Raman spectroscopy is also utilized to prove that there is no residual diamond films left on the tooth surfaces.

\section{Experimental Procedure}

\subsection{High density oxygen ashing process with the hollow cathode}

The original RF-DC oxygen plasma ashing system consists of the vacuum chamber, the plasma generator, the control unit, the carrier gas supply, and the plasma diagnosis instrument. The chamber is neutral in electricity. RF dipole electrodes and DC-bias work independently to generate RF and DC plasmas, respectively. Hence, the ionized species and activated radicals in the RF plasma are attracted to this DC biased plate with kinetic energy. Either RF-plasma or DC-plasma or, both are ignited by switching on either or both on the control panel. In addition, there is no mechanical matching box for RF plasma generation in this system. Both the input and output powers are automatically matched by frequency adjustment around $2 \mathrm{MHz}$. This automatic control significantly shortens the response time down to $1 \mathrm{~ms}$; hence, the oxygen plasma is available even in the mesoscopic pressure rage over $20 \mathrm{~Pa}$.

A standard experimental set up is summarized as follows. The base pressure is less than $5 \times 10^{-3} \mathrm{~Pa}$, and pure oxygen gas (purity; 99.99\%) is only used as a carrier gas. RF voltage, DC bias, and pressure are varied in a range from $60 \mathrm{~V}$ to $250 \mathrm{~V}$, from $-400 \mathrm{~V}$ to $-600 \mathrm{~V}$, and from $25 \mathrm{~Pa}$ to $100 \mathrm{~Pa}$, respectively.

A hollow-cathode device is also employed in this system to control the external plasma condition to ashing and to intensify the oxygen ion density enough to make complete and fast-rate ashing. As shown in Fig. 1, the generated RF-plasma is confined in this hollow so that higher ion and electron densities are attained for ashing the DLC- and diamond films (Yunata, et al., 2014; Yunata, et al., 2015; Yunata, et al., 2015; Yunata, et al., 2015). To be discussed in 
later, these ion and electron densities distribute in the hollow tube from its inlet of oxygen gas to outlet. Hence, where to place the diamond-coated tools becomes an issue to be solved by the quantitative plasma diagnosis in the inside of hollow. In the following the diamond-coated tooth part of tool is placed at the hot spot where the highest oxygen ion density state is attained. The same DC-bias is applied to both the hollow and the tool.

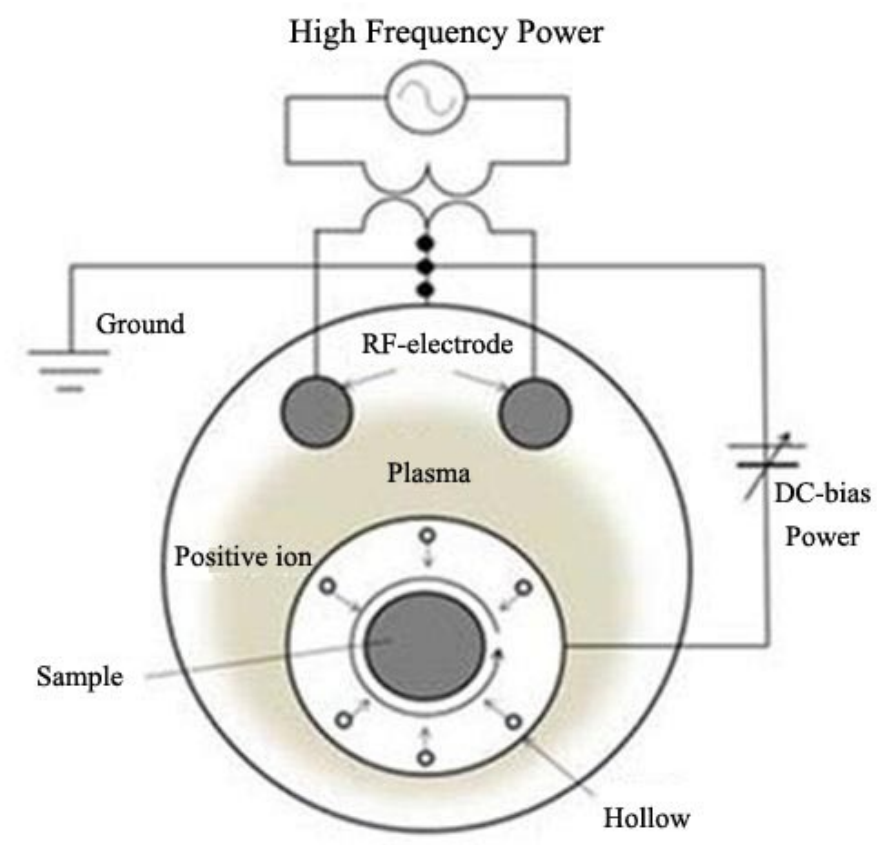

Fig. 1 The schematic of hollow cathode oxygen plasma ashing

Table 1 Plasma ashing experiment set up

\begin{tabular}{|c|c|c|c|c|}
\hline No. & RF-voltage (V) & DC-bias voltage (V) & Pressure (Pa) & Time (ks) \\
\hline $\mathbf{1}$ & 250 & 650 & 30 & 3.6 \\
\hline $\mathbf{2}$ & 250 & 500 & 45 & 3.6 \\
\hline $\mathbf{3}$ & 100 & 500 & 45 & 3.6 \\
\hline
\end{tabular}

Table 1 summarizes the experiment set up for plasma ashing process. Under this set-up, the tool is rotated with the constant rotational speed of 6 to 10 rotation per minute to make uniform ashing of diamond films on the whole tooth surfaces of tools. Then, the present ashing process is free from the shadow effect on the ashing; the diamond-coated back surfaces are easy to be removed.

\subsection{Quantitative plasma diagnosis}

Both the emissive-light optical spectroscopic (EOS) analyzer and the Langmuir probe were utilized to quantitatively describe the plasma state for ashing the diamond coatings. The former system (Hamamatsu Photonics, Col. Ltd) is useful to detect the activated species in wide scanning; e.g. activated oxygen atoms and molecules. In particular, this EOS-analyzer is useful as an in-process monitor to investigate the time variation of activated oxygen-peak and CO-peak intensities. The narrow scanning in the specified wavelength range is useful to trace the selected peaks with time.

The latter system (Impedance, Co., Ltd.) is also used to measure the relationship between the plasma current and the plasma voltage. This measured I-V curves are data-processed to deduce the ion and electron densities as well as the electron temperature at any position in the plasmas. 


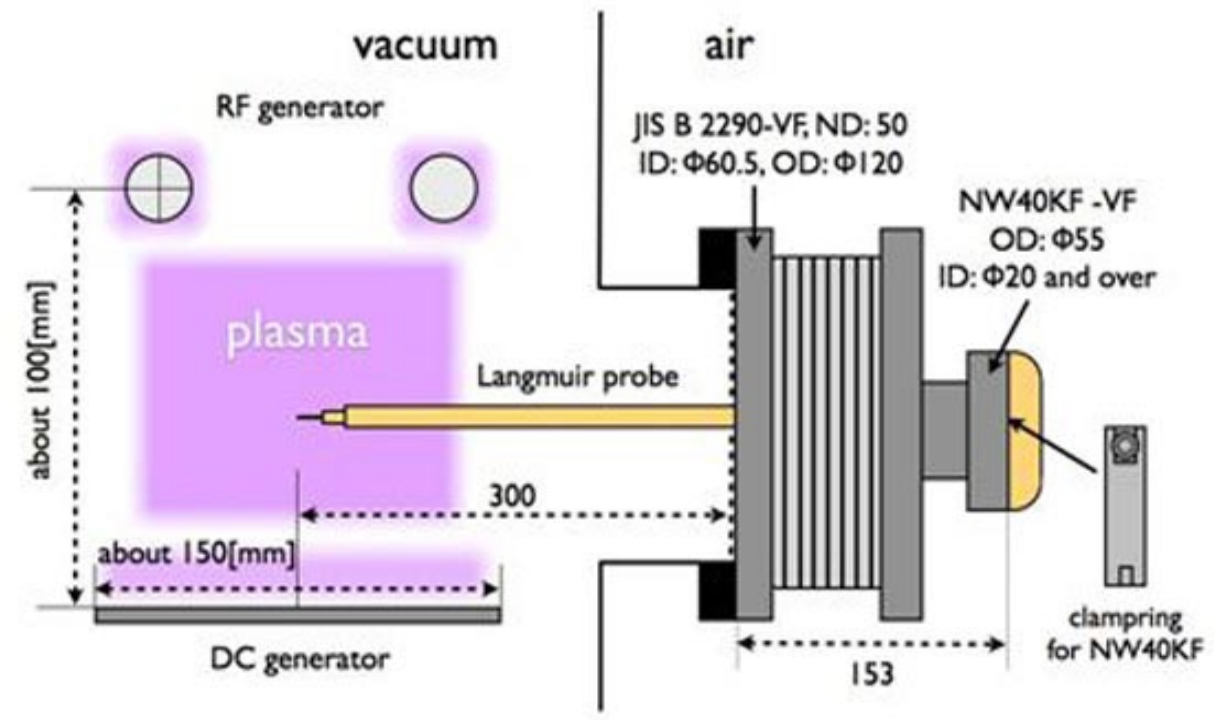

Fig. 2 The plasma density measurement by using Langmuir probe

In the present study, this Langmuir probe is driven to move itself in the inside of hollow along the direction of tools. Figure 2 depicts the experimental set-up of the Langmuir probe. Using the flexible bellows, the probe is controlled to move forward and backward in the hollow only by stepping the dials. In the following experiments, the origin in movement of probe, or, $\mathrm{x}=0$, is fixed to be $4 \mathrm{~cm}$ offset from the inlet of hollow and to measure the ion and electron density distributions from the inlet through the outlet of hollow tube.

\subsection{Specimen}

A CVD-coated end-milling tool with short shank was prepared for test-piece to investigate the ashing process by the present method. As shown in Fig. 3, the outer diameter of WC (Co) tool substrate was $10 \mathrm{~mm}$; its length, $70 \mathrm{~mm}$. This CVD diamond film was uniformly coated onto WC (Co) surface with the average thickness of $10 \mu \mathrm{m}$ after pretreatment. In this pretreatment, a part of cobalt binder in WC (Co) was chemically removed to build up the nucleation sites for enhancement of the diamond growth during coating. During the ashing experiments, the right-hand side shaft was fixed into the holder in the hollow cathode device in Fig. 1.

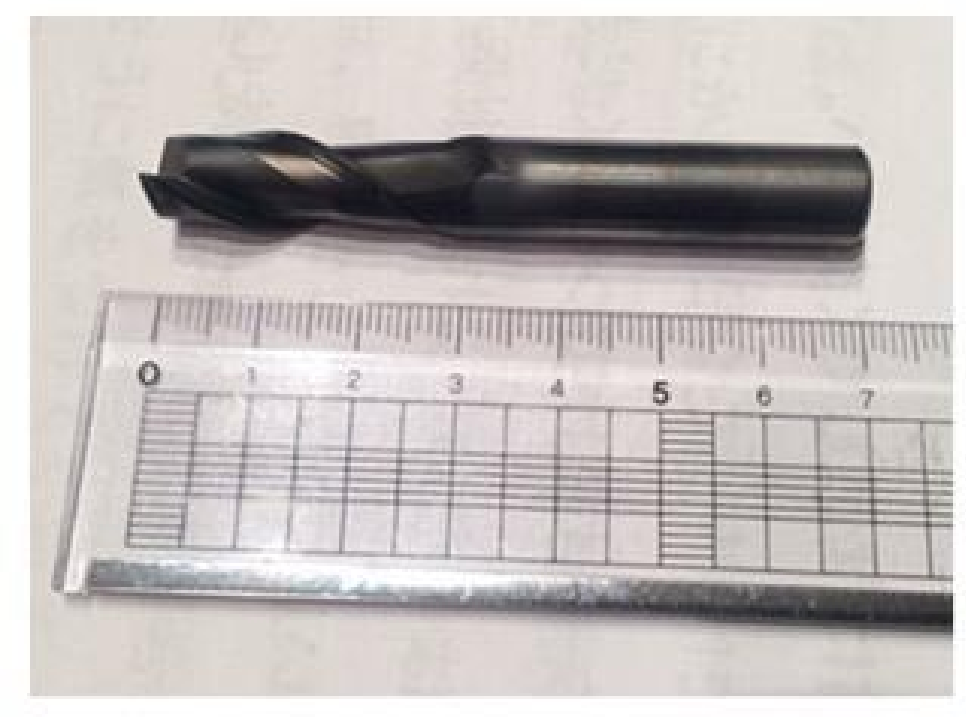

Fig. 3 CVD-diamond coated end milling WC (Co) tool specimen 


\subsection{Observation and Measurement}

SEM (scanning electron microscope; Nikon, Co. Ltd.) and optical microscope (Nikon, Co. Ltd.) were utilized to make micro-structural observation on the ashed diamond film surface. Raman microscopy (Renishaw, Co. Ltd.) was also used to prove that there were no residual diamonds on the WC (Co) substrate surface after ashing.

\section{Experimental Result}

\subsection{Plasma Diagnosis}

The plasma characteristic of the hollow cathode device is first described by the plasma diagnosis. Both the emissive-light optical spectroscopy (EOS) and the Langmuir probe were utilized to make quantitative diagnosis on the plasma state in the inside of the hollow cathode. In the spectroscopy, the detector was fixed on to the silica window with controlling the focus to the inlet of hollow. Figure 4 shows the measured spectra during the ashing process both in wide and narrow scanning, respectively. In the widely scanned spectrum, the activated oxygen atom or OI has the highest peak intensity at $\lambda=770 \mathrm{~nm}$. Through the enhanced ionization in the hollow device, much oxygen atoms are yielded in the hollow by the recombination reaction of $\mathrm{O}^{+}+\mathrm{e} \rightarrow \mathrm{O}$. Besides the high intensity peaks of OI, the ionized oxygen molecules (or $\mathrm{O}_{2}^{+}$) and the ionized oxygen atom (or OII, $\mathrm{O}^{+}$) are also detected with much lower peak intensities.

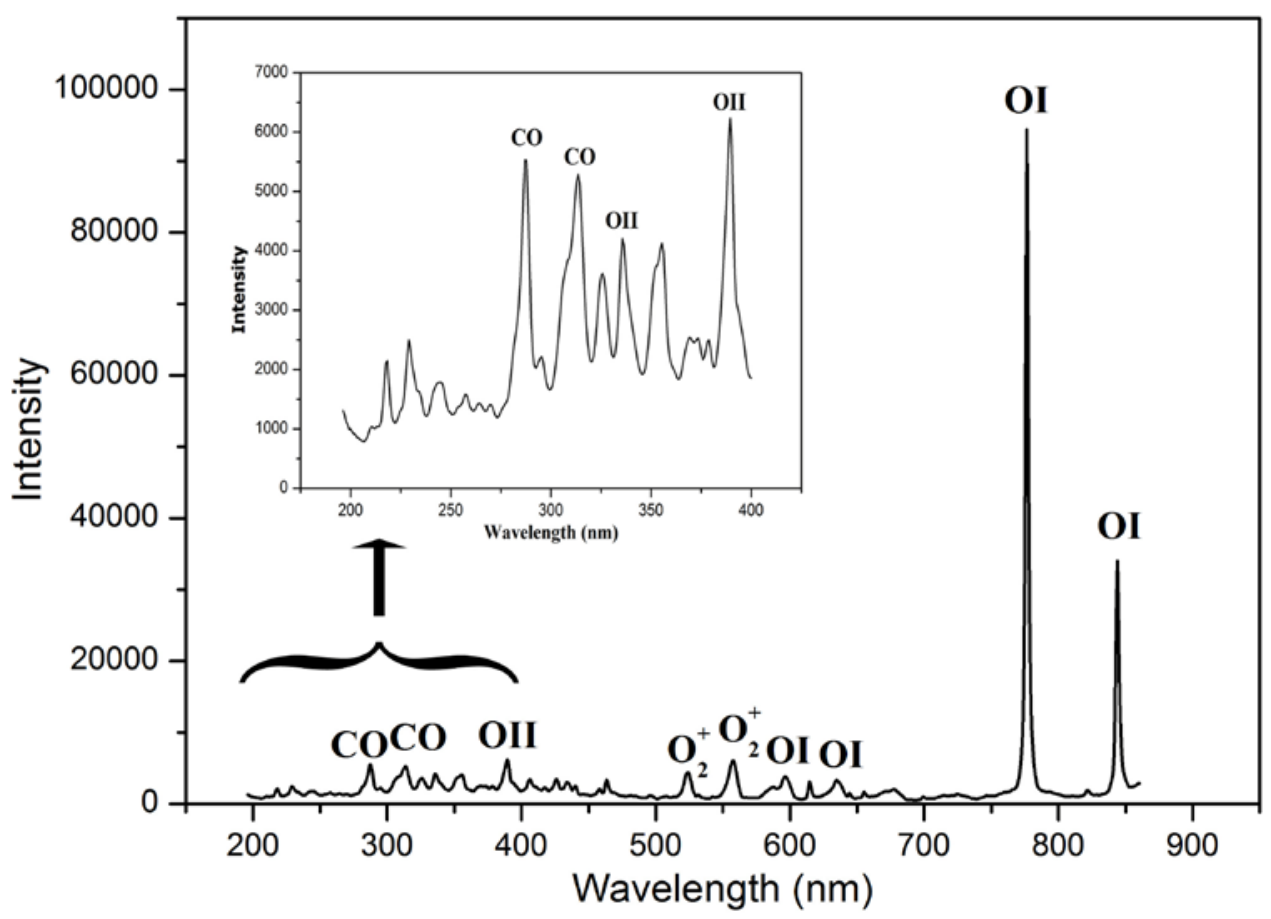

Fig. 4 The peaks identification during plasma ashing by using OES

In addition to the oxygen atoms and the ionized oxygen molecules and atoms, CO peaks are also detected even in the widely scanned spectrum. The insert figure in Fig. 4 shows the narrowed scanned spectrum in the wave length range $(\lambda)$ from $200 \mathrm{~nm}$ to $400 \mathrm{~nm}$. These CO peaks are detected at $\lambda=277 \mathrm{~nm}$ and $320 \mathrm{~nm}$, respectively. This implies that the diamond coating is ashed away by the direct reaction of $\mathrm{C}$ (in diamond) $+\mathrm{O}$ (oxygen flux from plasmas) $\rightarrow \mathrm{CO}$. Hence, this CO-peak becomes an indicator to describe the reactivity during the ashing process.

In this study, the Langmuir probe is controlled to move from the inlet of hollow to its outlet in the stepwise motion. Then, both the ion and electron densities distributions are directly obtained along the longitudinal direction of hollow, as respectively shown in Fig. 5. Since the origin $x=0$ is away from the inlet of hollow by $4 \mathrm{~cm}$ and the length of hollow is $8 \mathrm{~cm}$, the probe detects the variation of electron and ion density distributions in the inside of hollow tube from $\mathrm{x}=4$ to $12 \mathrm{~cm}$. 

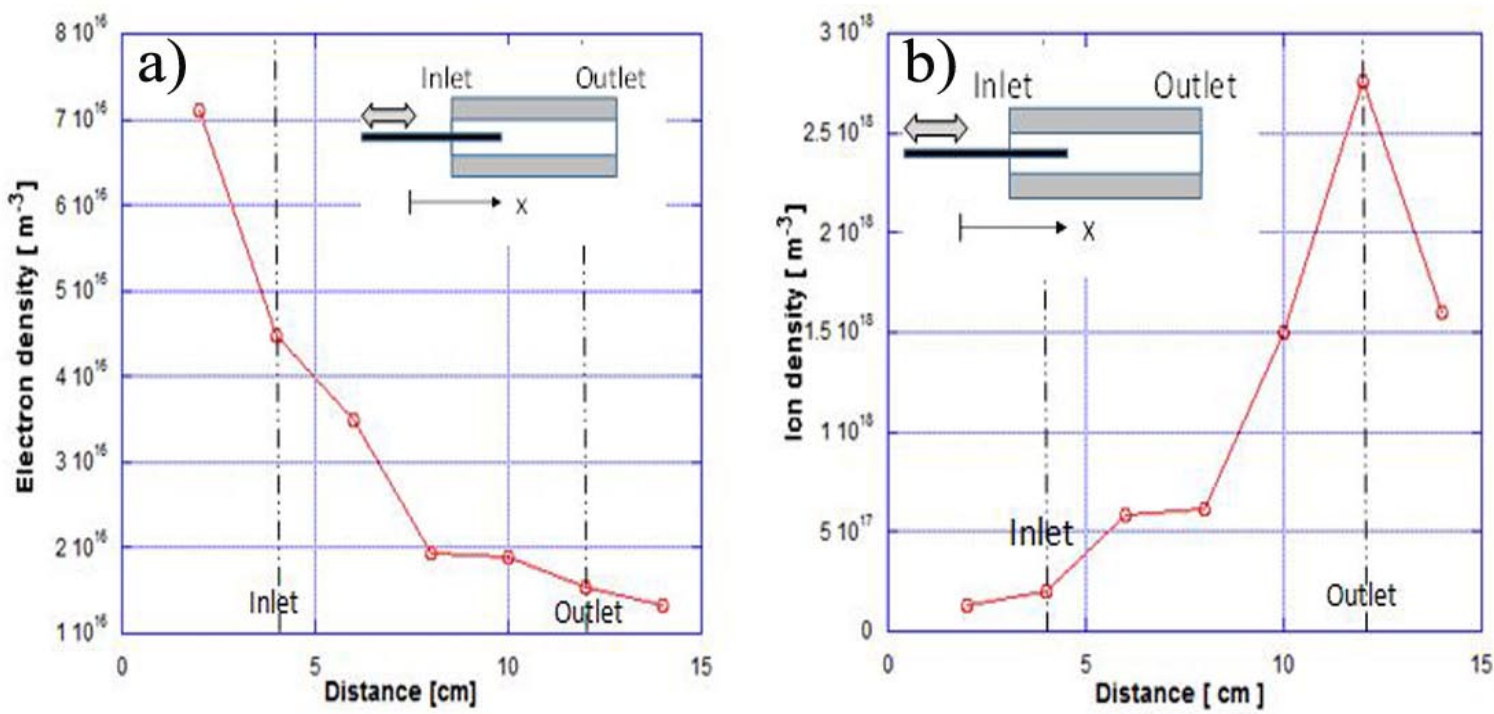

Fig. 5 The plasma density distribution from inlet to outlet of hollow cathode, a) electron density distribution and b) ion density distribution

As shown in Fig. 5 a), the electron density (Ne) remains to be around 5 to $7 \times 10^{16} \mathrm{~m}^{-3}$. This high Ne monotonically decreases in the $\mathrm{x}$-axis through the hollow. Most of electrons are recombined with the $\mathrm{O}^{+}$species to form the high oxygen atom flux at the hot spot with ionization. Figure $5 \mathrm{~b}$ ) depicts the oxygen ion density distribution in the $\mathrm{x}$-axis. Since the ionization is enhanced in the hollow cathode device, especially at the hot spot, the oxygen ion density increases exponentially from the inlet to the outlet of the hollow. In particular, the maximum ion density reaches to $3 \mathrm{x}$ $10^{18} \mathrm{~m}^{-3}$ at the vicinity of outlet. The oxygen ion density reported in (Aizawa, et al., 2013) was only $1 \times 10^{16} \mathrm{~m}^{-3}$ even for the inductively coupled oxygen plasmas. This hot spot with high ion density in the order of $10^{18} \mathrm{~m}^{-3}$ is suitable to make complete ashing of the diamond coatings on the tool substrate.

\subsection{Oxygen Plasma Ashing Processes}

Monitoring the time evolution of the CO-peak intensity at $\lambda=277 \mathrm{~nm}$ in Fig. 4, the ashing process is described under each specified processing condition. As shown in Fig. 6, high CO-peak intensity is detected in the initial stage up to $\mathrm{t}=0.5 \mathrm{ks}$; this corresponds to high reaction of carbon in diamond with the oxygen atom flux in the hollow by $\mathrm{C}$ (in diamond) $+\mathrm{O}$ (from plasmas) $\rightarrow$ CO. Since then, this CO-peak intensity gradually decreases with time; the reactivity gradually lowers with reduction of carbon sources of diamond films in time. Finally, the detected CO-peak intensity minimizes around $\mathrm{t}=3.6 \mathrm{ks}$; in the present study, the ashing process is commonly terminated at this time. Time variation of the O-peak intensities is also effective to describe the effect of processing parameters on the reactivity during ashing.

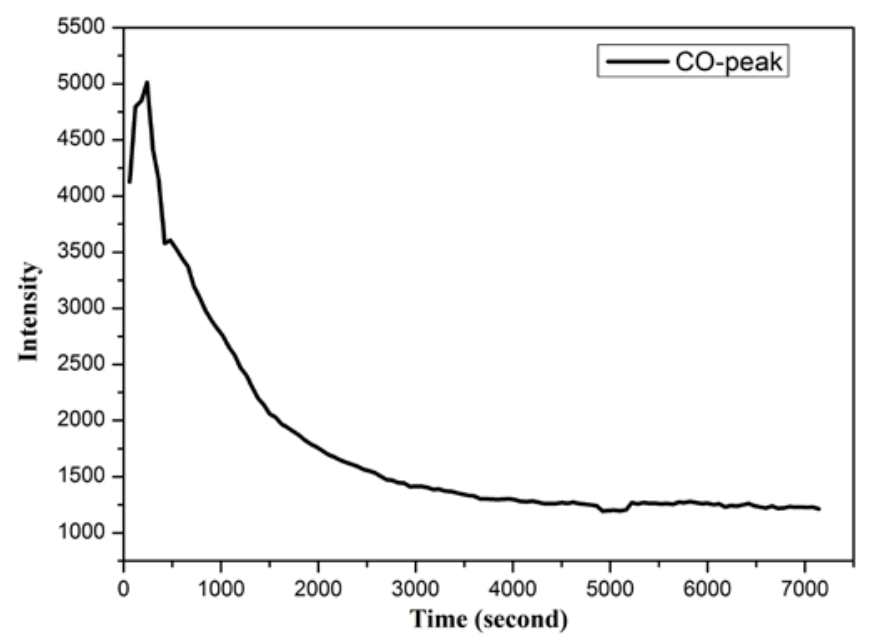

Fig. 6 Time evolution of CO peak again the time during plasma ashing process 


\subsection{Removal of Diamond Coating}

High ion density in the hollow cathode is utilized for ashing the diamond coatings from the WC (CO) tool substrate. The CVD-diamond coated, short-shank end-milling tool was employed as a test-piece for high density oxygen plasma ashing under the condition listed in Table 1. The optimum RF-voltage as well as DC-bias in Table 1 was searched by in-line monitoring the peak intensity of activated oxygen atoms through EOS to be discussed in later.

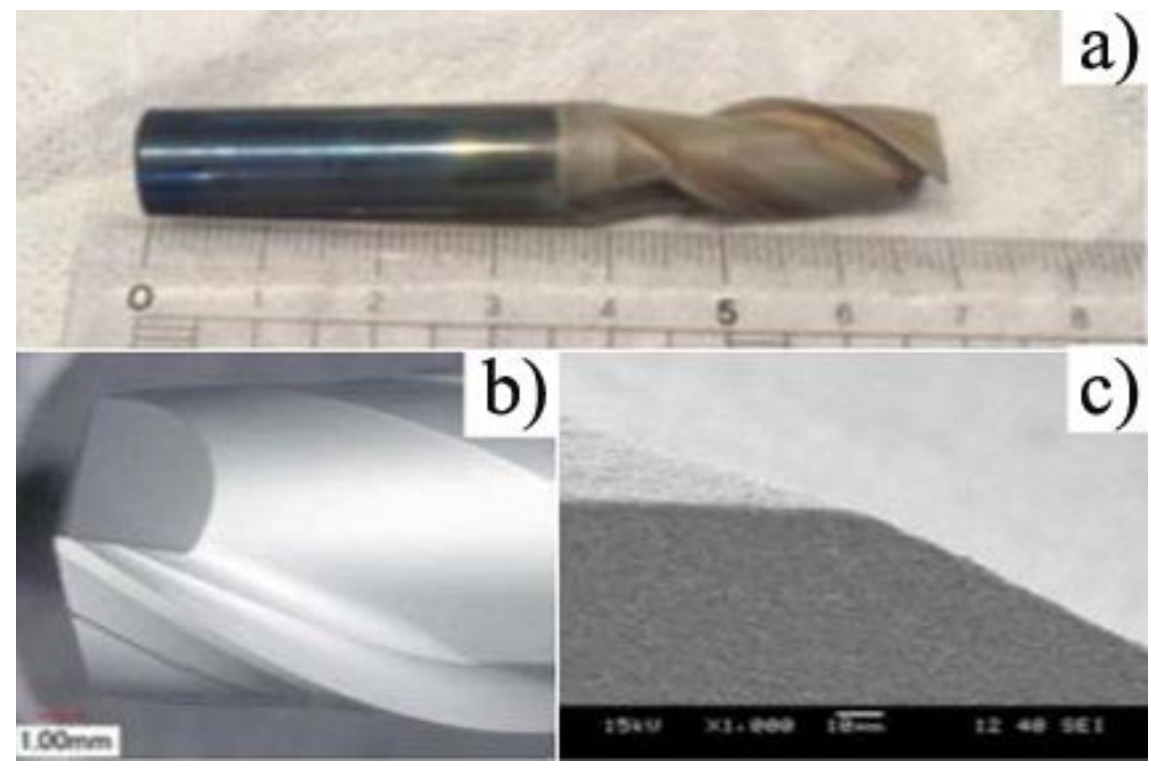

Fig. 7 CVD-diamond coated tool after ashing process, a) Outlook of ashed CVD-diamond coated tool, b) A top tooth of ashed tool after cleansing, and c) SEM image of tooth edge

Figure 7 a) shows the outlook of ashed tool surface. Although the original diamond coating is colored in black in Fig. 3, the ashed surface for $3.6 \mathrm{ks}$ appears to be light gray. After cleansing this gray dirt layer surface by the ultrasonic polisher, the original metallic WC (Co) surface appears as shown in Fig. 7 b). Figure 7 c) depicts the SEM image of the ashed WC (Co) tooth edge; the loss of WC (Co) by SEM in the tooth outer diameter is measured to be only $1.1 \mu \mathrm{m}$. This shrinkage of tooth diameter after ashing is much less than the engineering tolerance of $5 \mu \mathrm{m}$; this is suitable to the recycling of WC (Co) tool substrate in commercial.

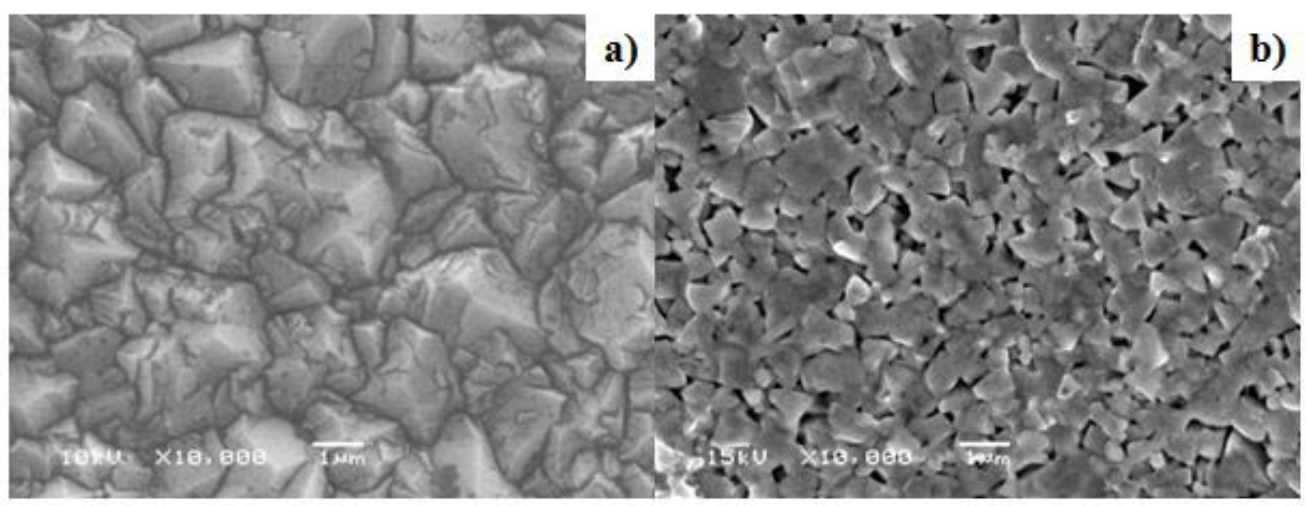

Fig. 8 The (a) cutting tool before ashing, and (b) after ashing process (return to the original surface)

The SEM image on the surface of diamond coating is compared before and after ashing in Fig. 8. The as-coated diamond film surface morphology is characterized by the rough, tetrahedral grains as depicted in Fig. 8 a). This is because of the three dimensional diamond grain growths from the arbitrarily distributed nucleation sites or the Co-debinded WC-grain-boundaries on the WC (Co) substrate surface. Figure 8b) shows the ashed surface of tooth surface for 3.6 ks. Tetrahedral grains as seen in Fig. 8a) disappears in the SEM image; the original Co-debinded 
WC-grain boundaries are seen among these WC grains. This proves that the original pretreated WC (Co) surface before diamond-coating, is recovered by ashing the whole diamond films. The Raman spectra are also compared in Fig. 9 before and after the present ashing process. In the Raman spectroscopy, the original diamond coating is characterized by the single, high-intensity peak around $\lambda=1330 \mathrm{~cm}^{-1}$. This characteristic spectrum completely disappears after ashing for 3.6 ks. This demonstrates that no diamond films are left even in local on the ashed surface of WC (Co) substrate.

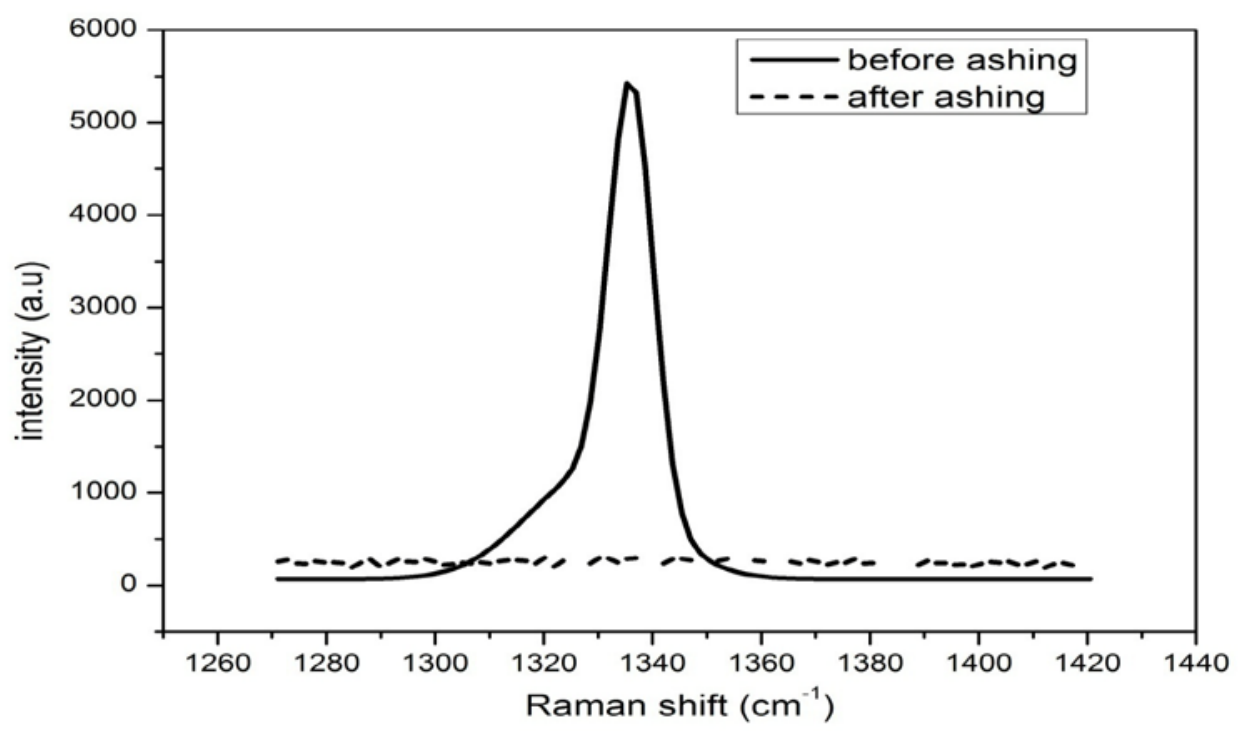

Fig. 9 Comparison of diamond peak before and after ashing by using Raman spectroscopy

\section{Discussion}

The hollow cathode device is characterized by enhancement of ionization from the oxygen molecules to oxygen ions. Through the spectroscopy, $\left\{\mathrm{O}, \mathrm{O}^{+}, \mathrm{O}^{2+}\right\}$ are detected as the oxygen species. After Refs. (Yunata, et al., 2014; Aizawa, et al., 2013; Cvelbar, et al., 2007), the supplied oxygen molecules are ionized to $\mathrm{O}_{2}^{+}$by electron detachment reaction of $\mathrm{O}_{2} \rightarrow \mathrm{O}_{2}^{+}+$e. This $\mathrm{O}_{2}^{+}$is ready to react with the electrons; $\mathrm{O}_{2}^{+}+\mathrm{e} \rightarrow \mathrm{O}^{+}+\mathrm{O}+$ e as the first electron attachment reaction. Since $\mathrm{O}^{+}+\mathrm{e} \rightarrow \mathrm{O}$, high peak intensities of $\mathrm{O}$ (or $\mathrm{OI}$ ) in Fig. 4 reveals that the oxygen atoms are generated as a main yield during this series of reactions in the highly ionized state by the hollow cathode device.

The Langmuir probe method reveals that the ionized oxygen ions are confined in the hollow tube and this results in high oxygen ion density. Assuming the oxygen carrier gas is statically supplied to this hollow, the oxygen ion density (Ni) distributed in the Gaussian profile, where Ni becomes maximum at the center of hollow and reduces itself to both ends. As shown in Fig. 5, the carrier gas flows into an inlet of hollow through a nozzle; the density profile is shifted to the outlet end of hollow. On the other hand, the electrons are easy to run away from the hollow along the magnetic force lines. The electron density becomes lower in the inside of hollow.

As the first step to fast-rate and homogeneous ashing, the end-milling tooth part is placed at the hot spot with highly activated oxygen ion density in Fig. 5. The either end of tool specimen is fixed to the jig, through which the same DC-voltage as the DC-bias is applied to the tool. The oxygen gas flows from the inlet of hollow, through the narrow clearance between the inner hollow surface and tool surface, and goes out of the other end of hollow. This spatial configuration in the experimental set-up is necessary to attain the fast-rate, homogeneous ashing state even for various kinds of used diamond-coated tools with different tooth geometry, size and dimension.

In second, the rotational control of end-milling tool is indispensable to make homogeneous ashing of diamond films. In fact, when fixing the tool specimen without rotation, or, when rotating it with higher speed, the residual diamond films were left on the back of tooth surfaces or at the tooth edge.

To minimize the loss of WC (Co) tooth geometry and dimension in the ashing process, over-oxidation of WC (Co) must be saved by optimization of RF-voltage and DC-bias. As had been investigated in (Yamauchi, et al., 2015), the RF-voltage (or $\mathrm{V}_{\mathrm{RF}}$ ), the DC-bias (or $\mathrm{V}_{\text {bias }}$ ) and the pressure (P) were varied to search for an optimum ashing condition. As told before, the in-line monitoring of detected CO-peak intensity works well to understand the reactivity condition during ashing. In parallel with this, the in-line monitoring of activated oxygen atom peak intensities provides a 
direction to be free from over-oxidation of WC (Co) tool teeth. When $\mathrm{V}_{\mathrm{RF}}=250 \mathrm{~V}, \mathrm{~V}_{\text {bias }}=-650 \mathrm{~V}$, and $\mathrm{P}=30 \mathrm{~Pa}$, the ashed tooth surface turns to be yellow or green; tungsten in WC (Co) is oxidized to be $\mathrm{WO}_{2}$ or $\mathrm{WO}_{3}$. In this case, the activated oxygen peak intensity significantly reduces itself for $\mathrm{t}>2 \mathrm{ks}$ in the in-line monitored EOS. Figure 6 proves that most of diamond films are removed for $\mathrm{t}>2 \mathrm{ks}$ and that reactivity of $\mathrm{C}$ (in diamond) $+\mathrm{O}$ (from plasmas) $\rightarrow \mathrm{CO}$ reduces itself. Hence, this significant reduction of oxygen atoms in the latter half of ashing duration corresponds to the over-oxidation of WC (Co). When lowering the $\mathrm{V}_{\mathrm{RF}}$ to $100 \mathrm{~V}$ and the $\mathrm{V}_{\text {bias }}$ to $-500 \mathrm{~V}$, respectively, the tooth surface becomes light gray and there is no residual diamond films after ultrasonic cleansing in Fig. 7a) and 7b). In this case, the oxygen atom peak intensity remains to be the same as the initial intensity without any reduction. This proves that quality of WC (Co) substrate surface and geometry during the ashing process is preserved by optimization of the process parameters and that in-line monitoring of activated oxygen atom peak intensity by EOS provides a means to preserve high quality assurance.

\section{Conclusion}

High density oxygen plasma ashing with use of the hollow cathode device is effective to make perfect removal of CVD-diamond coatings in short processing. Termination of the present ashing process is determined by the minimization of the detected CO-peak intensity in the in-line monitoring of EOS spectra. The diamond film with the thickness of $10 \mu \mathrm{m}$ is ashed away by $1 \mathrm{~h}$ or $3.6 \mathrm{ks}$; the average ashing rate is $10 \mu \mathrm{m} /$ hour or $3 \mu \mathrm{m} / \mathrm{ks}$, twenty times faster than the commercial processes. Both RF-voltage and DC-bias are varied to search for the ashing process conditions to minimize the damage of WC (Co) tooth of tools. With the RF-voltage of $100 \mathrm{~V}$ and the DC-bias of -300 $\mathrm{V}$, the reduction of outer tooth diameter for WC (Co) tool becomes $1 \mu \mathrm{m}$, less than the design standard necessary for recycling the WC (Co) tool substrate by 1/5. Raman spectroscopy proves that no diamond residuals are left on the ashed tooth surfaces. FE-SEM also demonstrates that the decobalted microstructure on the WC (Co) tool surface is recovered by the present ashing; the pre-treated WC (Co) tooth surface is to be used for recoating the diamond film on it.

\section{Acknowledgements}

The authors would like to express their gratitude to Mr. Y. Sugita (YS-Electric Industry, Co. Ltd.) for precise discussion. The present study is financially supported in part by MEXT and by JST, respectively. 


\section{References}

Aizawa, T., Masaki, E., Sugita, Y., Oxygen plasma ashing of used DLC coating for reuse of milling and cutting tools, Proc. Int. Conf. Mater. Process, (MAPT-2013), (2013, BKK), pp.15-20.

Aizawa, T., Masaki, E., Sugita, Y., Complete ashing of used DLC coating for reuse of the end-milling tools, Manufacturing Letters, 2, (2014), pp. 1-3.

Aizawa, T., Masaki, E., Morimoto, E., Sugita, Y., Recycling of DLC-coated tools for dry machining of aluminum alloys via oxygen plasma ashing, Mechanical Engineering Research, 4, (1), (2014), pp. 52-62.

Aizawa, T., Mizushima, K., Redationo, T. N., Yang, M., Micro-imprinting onto DLC and CNT coatings via high density oxygen plasma etching, Proc. 8th ICOMM Conference (2013, Canada, Victoria), pp. 459-466.

Aizawa, T., Sugita, Y., High-density plasma technology for etching and ashing of carbon materials, Res. Rep. SIT, 55-2, (2011), pp. 13-22.

Aizawa, T., Sugita, Y., High density oxygen plasma ashing for recycling and reuse of DLC-coated tools and dies, Res. Rep. SIT 58-1 (2014), pp. 17-26.

Allen, D. M., et. al., Ion beam, focused ion beam, and plasma discharge machining, Manufacturing technology, 58, (2009), pp. 647-662.

Cvelbar, U., Krstulovi, N., Miloševi, S., Mozeti, M., Inductively coupled RF oxygen plasma characterization by optical emission spectroscopy, Vacuum, 82, 2, (2007), pp. 224-227.

Gangopadhyay, S., Acharya, R., Chattopadhyay, A. K., Sargade, V. G., Effect of cutting speed and surface chemistry of cutting tools on the formation of burrs and surface quality of the generated surface in dry turning of AA6005 aluminum alloy, Machining Science Technology, 14, 2, (2010), pp. 208-223.

Hasegawa, R., Cutting tool for aircraft and applications, Journal of Japan Society for Precision Engineering, 75, 8, (2009), pp. 953-957.

Kurita, T., et. al., Advanced material processing with nano- and femto- second pulsed laser, International journal of machine tools and manufacture, 48, (2008), pp. 220-227.

Syrkin, A., et. al., Reactive ion etching of $6 \mathrm{H}-\mathrm{SiC}$ in an ECR plasma of CF4-O2 mixture using both $\mathrm{Ni}$ and $\mathrm{Al}$ mask, Materials science and engineering, B46, (1997), pp. 374-378.

Yamauchi, K., Yunata, E. E., Aizawa, T., High density oxygen plasma ashing of CVD diamond coating for recycling of WC (Co) tools, Proc. $4^{\text {th }}$ IFMMBF-2015, (2015, May, Toyama), pp. 33-36.

Yunata, E. E., Aizawa, T., Djoko Santjojo, D. J. H., Characterization of hollow cathode plasma for etching and ashing processes, Proc. 8th SEATUC Conference (2014, UTM, Johor Balu, Malaysia), CD-ROM.

Yunata, E. E., Aizawa, T., Micro-texturing into DLC/diamond coated molds and dies via high density oxygen plasma etching,Manufacturing Review 2 (2015), pp. 1-8.

Yunata, E. E., Aizawa, T., High density oxygen plasma micro-texturing into CVD-diamond coated dies for micro-embossing, Proc. 10th 4M/ICOMM2015. (2015), CD-ROM.

Yunata, E. E., Aizawa, T., High density oxygen plasma processing for ashing of DLC and CVD diamond Coated tools, J. Coating. (2015), (to be published).

Zolgharni, M., Jones, B. J., Bulpett, R., Anson, A. W., Franks, J., Energy efficiency improvements in dry drilling with optimized diamond-like carbon coatings, Diamond and Related Materials, 17, (2008), pp.7-10. 